\title{
LINEAMIENTOS PARA EL DISEÑO DE LA INTRANET DE UNA ESCUELA DE NEGOCIOS. CASO: ENV
}

\author{
Sandra Orjuela-Córdobaํ. Universidad Monteávila. Venezuela \\ sandraorjuelacordoba@gmail.com
}

\section{RESUMEN}

Las nuevas tecnologías al servicio de las organizaciones, han generado nuevas formas de trabajo, metodologías y perfiles profesionales, lo que ha llevado a las universidades a replantearse sus paradigmas de gestión y comunicación. Dentro de estos cambios y nuevas tendencias, las Universidades son escenarios idóneos para reflexionar, profundizar y experimentar en cuanto a la eficacia y eficiencia de los medios como la Intranet, dentro de un plan de comunicación. La paradoja que se presenta aquí, es que la Universidad por un lado es el ente donde se investiga, comparte y debate sobre el tema y por el otro en su propia aplicación puede presentar serios inconvenientes, a la hora de aplicar esos conocimientos dentro de la misma institución. El objetivo principal de este trabajo es diagnosticar la efectividad de la Intranet como medio de comunicación interna, específicamente aplicado a una Escuela de Negocios en Venezuela. (145).

PALABRAS CLAVE: Universidad - Intranet - Diagnostico - Plan - Comunicación

\footnotetext{
${ }^{1}$ Autor correspondiente

Sandra Orjuela Córdoba: Profesora e investigadora en la Universidad Monteávila. Caracas (Venezuela). Correo: sandraorjuelacordoba@gmail.com
} 


\title{
GUIDELINES FOR THE DESIGN OF AN INTRANET BUSINESS SCHOOL. CASE: ENV
}

\begin{abstract}
New technologies for organizations, have created new forms of work, methodologies and professional profiles, which has led universities to reconsider their paradigms of management and communication. Within these changes and new trends, the universities are ideal scenarios for reflection, depth and experience regarding the effectiveness and efficiency of the media as the Intranet, in a communication plan. The paradox here is that the University on one side is the body which investigates, share and debate on the subject and the other in your own application can have serious drawbacks when applying this knowledge within the same institution. The main objective of this work is to diagnose the effectiveness of the Intranet as a means of internal communication, specifically applied to a business school in Venezuela.
\end{abstract}

KEY WORDS: University - Intranet - Diagnosis - Plan - Communication

\section{INTRODUCCIÓN}

Las nuevas tecnologías al servicio de las organizaciones, se han convertido en un tema de permanente actualidad, donde el vertiginoso desarrollo de éstas ha sido más avanzado que el estudio, análisis y reflexión acerca de sus beneficios y limitaciones para las empresas, así como de su papel en el proceso de comunicación exitosa.

Para, Fernando Núñez las tecnologías de la información y la comunicación, han producido cambios en la Comunicación Social, generando nuevas formas de trabajo, metodologías y perfiles profesionales. Tanto así que se han creado "nuevos mundos" como la escritura multimedial, la blogósfera, las redes sociales, el internet telefónico y el denominado "periodismo ciudadano", entre otros. (Núñez, 2009, p.24)

Debido a esta nueva realidad, se propone un "esquema digital de la comunicación (EDC), una aproximación que contraste los modelos tradicionales de comunicación interpersonal y masiva con los cambios digitales mencionados" (Núñez, 2009, p.27).

Estos nuevos paradigmas enfrentan al comunicador organizacional al reto de lograr una simbiosis entre los medios tradicionales y las nuevas tecnologías, adecuando su concepción, conocimientos, experiencias y planes a la realidad organizacional en el plano digital y tradicional, para garantizar la consecución de sus objetivos 
Dentro de estos cambios y nuevos paradigmas las Universidades son agentes muy importantes para reflexionar, profundizar, dar a conocer y experimentar en cuanto a la eficacia y eficiencia de los medios como la Intranet, dentro de un plan de comunicación. La paradoja que se presenta aquí es que la Universidad por un lado es el ente donde se investiga, comparte y debate sobre el tema y por el otro en su propia aplicación puede presentar serios inconvenientes, a la hora de aplicar esos conocimientos dentro de la misma institución.

\section{METODOLOGÍA}

La Intranet, como medio de comunicación interna en una institución universitaria es el tema de este trabajo, donde se busca diagnosticar la efectividad de este medio en el proceso comunicacional de una Escuela de Negocios, que hace parte de una prestigiosa universidad en Venezuela. En adelante le denominaremos ENV.

Inicialmente se definirá el papel de la Intranet en el plan de comunicaciones internas, seguido por la identificación de las características técnicas y de contenido con las que cuenta esta Intranet, para posteriormente mostrar la opinión que los empleados de la ENV tienen frente a este medio de comunicación interna de la institución.

La metodología utilizada es el análisis de contenido a partir de una plantilla elaborada específicamente para este caso. Para darle contexto al estudio, se acompañó de las técnicas de entrevistas semi-estructurada a las directivas (2) con el objeto de definir el papel de la Intranet en el plan de comunicaciones, así como conocer cómo fue concebida, sus objetivos y expectativas respecto a ella y además se aplicaron $14(14 / 20)$ encuestas a los empleados de la ENV. Mediante estas encuestas se busca conocer su opinión acerca de la efectividad comunicacional de la Intranet, así como lograr una visión panorámica acerca del estado de dicha comunicación, pues no se puede desligar al medio del contexto donde se desarrolla.

La técnica de observación directa permitió analizar la Intranet a través de un instrumento diseñado para este estudio y que cuenta con 57 variables de análisis, divididas en nueve categorías además de un espacio para observaciones generales, lo que permitió identificar sus características técnicas, además de poder obtener datos sobre sus contenidos específicos, desde el punto de vista de ser una institución universitaria enfocada a los programas de cuarto nivel. Así mismo se pretende identificar si cuenta con herramientas para su manejo de prensa. Se presenta cada variable con la opción sí o no, para determinar si existe o no dentro del sitio.

Este instrumento fue diseñado a partir de la amplia lectura y comprensión previa de literatura especializada, acerca de las características que debe tener un sitio web y puntualmente una Intranet que garantice que el usuario encuentre con facilidad la información que busca, sea fácil de usar y ofrezca opciones de interactividad, lo que 
lo llevaría a ser un sitio exitoso en su labor de optimizar la comunicación y relacionamiento interno, entre los miembros de la ENV.

En el primer bloque se registra la información general concerniente a la Intranet, cuyo nombre en la ENV es: Sistema Automatizado de Administración Integral (SAAI), se revisa si hay relación entre la identidad visual de la ENV y la Universidad, ubicación de la marca visual, si posee presentación de la Directora, si tiene segmentación de públicos, cuenta con boletines electrónicos, hay sección de noticias de la Escuela de Negocios o de la Universidad, si hay opciones de ocio, se puede personalizar y si la información está actualizada.

En la segunda categoría, se identifican las características de usabilidad de la Intranet, a partir de la organización de la información por categorías y menús, la marca visual y los títulos identificadores de contenido en cada pantalla, herramientas de ubicación dentro del sitio (dónde está, de dónde viene, adelante, atrás, volver, seguir) y aplicaciones que informen al usuario si están realizando algún proceso y deben esperar, como por ejemplo: buscando, guardando, enviando, etc.

El tercer apartado registra la autonomía y control del sitio, a través de las variables: mapa de sitio, rutas de navegación, facilidad de volver a menús anteriores y de salir del sistema, así como la existencia de un motor de búsqueda al interior del sitio y conocer si es fácil de navegar.

La cuarta categoría es legibilidad, en la cual se registra si el tipo de letra es legible, utiliza frases y párrafos cortos, los íconos en conjunto son visual y conceptualmente claros y si el contraste entre texto y fondo es equilibrado y permite la lectura.

En el quinto apartado, se levanta información sobre la consistencia del diseño al revisar si la identificación de páginas y secciones es uniforme, al llegar por azar a una página se distingue claramente en qué lugar se encuentra, los comandos se utilizan de la misma manera en todo el sistema y se finaliza revisando si se respetan las convenciones como por ejemplo el sombreado de los vínculos ya visitados.

En la sexta categoría, las variables se refieren a la eficiencia del sistema analizando tiempo de carga del home, número de clicks (pinchar) para llegar a la información que se busca, si la interfaz ofrece varias opciones para buscar la información, formas para manejar la información (descargas, envío por mail, sólo texto, imprimir, envío a otro miembro de la ENV), si la estructura del sitio es simple y además si el usuario tiene que recordar permanentemente las reglas de uso propias del sitio.

La séptima variable recoge la existencia de medios interactivos, como: foros, chat, blog, RSS, twitter, facebook y si tiene tutorial o ayuda en línea. 
El octavo apartado está dedicado a la información especializada sobre la ENV, de acuerdo a su dinámica como centro de ofertas académicas para niveles gerenciales en el país. Se espera que presente como un mínimo de contenidos: información general de la ENV (filosofía, manuales de funciones, de procesos, directorios, memorias, documentos), tutoriales. Información general de la Universidad a la cual pertenece esta Escuela, información sobre los cursos que se ofrecen al público externo, información sobre profesores, bases de datos, link con la Universidad y sus aliados y sección de preguntas frecuentes.

La definición de las variables que se deberían tener en cuenta en este octavo apartado, fueron definidas a partir de entrevistas informales con un experto en comunicación digital, un asesor en Comunicación Corporativa y Branding Corporativo y una prestigiosa directora de prensa y comunicaciones de una

Universidad venezolana. Vale la pena aclarar que los 3 expertos son comunicadores sociales.

En el tipo de información que se considera debe tener esta Intranet Universitaria, no se tiene en cuenta espacio puntual para la necesidad de profesores y alumnos, porque después de la entrevista inicial con la Directora de Mercadeo y líder de la Intranet, se explicó que eso hace parte de una segunda etapa a mediano y largo plazo.

El noveno apartado se dedica exclusivamente a la sala de prensa virtual y para ello se pregunta: si existe una sala de prensa virtual en el sitio, en caso de ser positiva la respuesta se prosigue registrando si existe un archivo de notas y comunicados de prensa, archivo de fotos, archivo de videos y agenda de eventos.

Se termina con un espacio para observaciones generales, donde se colocan datos puntuales de interés a tener en cuenta para el estudio en cuestión, como en esta ocasión las secciones con las que cuenta la Intranet o SAAI.

\section{ANÁLISIS Y DISCUSIÓN}

\subsection{La Intranet y la comunicación organizacional}

El comunicador no debe olvidar en ningún momento que los medios deben utilizarse como vínculos entre individuos y no sólo como relaciones entre máquinas, programadores y usuarios, lo que afecta el resultado de la comunicación.

El desarrollo actual de un plan estratégico de comunicaciones para una organización, no puede olvidar el uso e impacto de los medios digitales para hacer llegar información de forma oportuna y segmentada, así como abrir espacios para la participación de todos los miembros que la componen. 
Es importante entender que el utilizar medios digitales, no implica que los públicos con quienes interesa comunicarnos, realmente reciben el mensaje y lo entienden. Uno de los retos actuales es encontrar la forma de medir y verificar que realmente el uso de nuevas tecnologías está mejorando el proceso de información y sobre todo está generando una optimización de la comunicación, mejorando la productividad, los procesos organizacionales y las relaciones entre los diferentes miembros.

El comunicador debe conocer y entender las características de cada medio digital, para poder utilizarlos en su justa medida dentro del plan estratégico de comunicaciones. Es importante que se implique en el proceso de diseño de los medios digitales y no lo deje sólo a un programador o ingeniero de sistemas, pues sólo el comunicador conoce y entiende qué medios debe utilizar para qué públicos, en qué momento, para transmitir qué tipo de mensaje y buscando qué efecto. Uno de los errores más comunes que se encuentran en la empresa es la multiplicidad de medios digitales como son: correo electrónico, sitios Web e Intranet, Mensajería Instantánea, telefonía: móvil y la IP, así como algunos servicios asociados².

Por ejemplo la Intranet, éste medio se ha hecho muy famoso en las organizaciones, pero es común encontrar que se crea que el hecho de tenerla garantiza una información y comunicación, veraz, eficiente, oportuna y esto sólo se logra si hace parte un plan estratégico de comunicación. En caso contrario, sólo se convertirá en un administrador de datos y no podrá garantizar el objetivo comunicacional para el cual debe ser desarrollada, además de ser apoyo en la generación de conocimiento para la gestión de los procesos y funciones de la institución.

El Dircom o responsable de la comunicación en la organización debe entender que la gestión de los medios digitales implica un trabajo interdisciplinario, para su adecuado y exitoso uso.

Sergio Llano, estima que desde el punto de vista estratégico, la comprensión del comunicador acerca del tema digital, puede darle perspectiva de cómo alinear una condición tecnológica en la búsqueda de una meta de comunicación organizacional y éste conocimiento incluso, podría permitirle en el tiempo, una capacidad para juzgar el entorno de las TIC, que le permita tener visibilidad en un equipo directivo y que contribuya indirectamente a la toma de decisiones estratégicas en la organización. (Llano, 2007, p.198)

\footnotetext{
${ }^{2}$ Caso de una empresa del sector financiero en Venezuela que concentró sus esfuerzos en los medios digitales, desarrollando 7 medios (además de otros medios impresos y audiovisuales) y al realizar un diagnóstico de sus sistemas de información v comunicación, el $86 \%$ de los públicos internos
} 
A partir de las explicaciones de Llano, acerca de las características según la naturaleza de los medios digitales y tradicionales, se ha construido el siguiente cuadro para facilitar la comprensión a la hora de diseñar medios, mensajes y estrategias por parte del equipo de comunicación en una empresa (Llano, 2007):

Tabla 1. Características hora de diseñar medios, mensajes y estrategias de comunicación

\begin{tabular}{|l|l|}
\hline \multicolumn{1}{|c|}{ Nuevos Medios } & \multicolumn{1}{c|}{ Medios Tradicionales } \\
\hline Digitales & $\begin{array}{l}\text { Análogos, sobre todo en su forma } \\
\text { de consumo }\end{array}$ \\
\hline $\begin{array}{l}\text { Permiten la personalización de } \\
\text { contenidos }\end{array}$ & $\begin{array}{l}\text { Tratan a sus audiencias sin } \\
\text { distinciones. }\end{array}$ \\
\hline Medios convergentes & Medios divergentes \\
\hline $\begin{array}{l}\text { Los lenguajes se integran } \\
\text { generando una condición de } \\
\text { convergencia con implicaciones } \\
\text { sustanciales en los procesos de } \\
\text { comunicación le }\end{array}$ & $\begin{array}{l}\text { Desde el punto de vista del } \\
\text { lenguaje, por ejemplo la prensa, la } \\
\text { radio y la televisión conservan } \\
\text { ámbitos propios e } \\
\text { independientes }\end{array}$ \\
\hline $\begin{array}{l}\text { Permiten la no linealidad en el } \\
\text { acceso a los contenidos }\end{array}$ & $\begin{array}{l}\text { Los contenidos se entregan de } \\
\text { forma secuencial }\end{array}$ \\
\hline
\end{tabular}

Es importante que el comunicador mida en su justa medida el beneficio y virtudes de la utilización de las TIC y de los medios digitales, para evitar fracasos en la planificación de comunicaciones. Podrá apoyarse en estos medios, pero no sujetar el plan sólo a su uso, pues aún falta mucho camino por recorrer en la inclusión de todos los ciudadanos en los ambientes digitales.

\subsection{La Planificación de la Intranet}

Una Intranet se puede considerar como una Internet interna diseñada para ser utilizada dentro de los límites de una compañía, universidad u organización. Lo que distingue una Intranet de la Internet libremente accesible, es que las Intranets son privadas.

Son varias las definiciones y elementos que se le atribuyen a la Intranet, por lo cual es importante en el momento de diseñar una herramienta de este tipo para la organización, tener en cuenta algunos aspectos mínimos que garanticen su papel como medio efectivo de comunicación interna. Al respecto Inmaculada Postigo, señala que: 
Es una red interna de una organización: es decir, se hace especial hincapié en dos conceptos. De una parte el concepto de red, de interacción, de posibilidad de contacto entre todos sus puntos de manera fácil y dinámica. De otra, el calificativo de interna, es decir, sus usuarios son todos aquellos que en el plan de comunicación estén caracterizados como públicos internos de la organización.

Utiliza las tecnologías de internet: la informática y la conexión de diversas estaciones es condición indispensable. De hecho, muchas de las definiciones dadas por algunos investigadores, únicamente inciden en este aspecto, que, a nuestro entender, sí que es un requisito fundamental, pero no el único para que algo sea considerado una intranet.

Permite compartir información, comunicación y colaboración: una intranet no es únicamente un lugar donde "colgar" información, aunque sirve para ello, una intranet no es sólo un lugar que permite la comunicación rápida y ágil entre los distintos usuarios a través del correo $u$ otros sistemas, aunque sirve para ello, y una intranet no es sólo un lugar dónde realizar tareas de trabajo de manera conjunta, aunque también sirve para ello. Una intranet es el conjunto de estas tres cosas, y si alguna de ellas no es utilizada, se está perdiendo mucho de su potencial.

Su función es respaldar la comunicación interna y por derivación, ayudar a conseguir un óptimo funcionamiento de la compañía:... es la intranet la que debe estar al servicio de la comunicación y no al contrario. (Postigo, 2004, p.34)

Las nuevas tecnologías se han ido convirtiendo falsamente en los paladines de salvación para las organizaciones. Se tiene la visión por parte de muchos directivos y coordinadores de diversas áreas, incluidos en algunos casos los directores de comunicación, que con tener un mix de medios digitales ya se garantiza la efectividad e intención de los mensajes y garantiza el logro de los objetivos comunicacionales. Esta situación representa un reto para los líderes de comunicación organizacional, ya que son los profesionales que cuentan con el conocimiento de la ciencia de la comunicación lo que les permite planificar, desarrollar, hacer seguimiento y evaluar todas las actividades de acuerdo a los objetivos y perfiles comunicacionales de los públicos internos y externos, además de convertirse en unos evangelizadores en los temas de comunicación a nivel transversal dentro de su institución.

El comunicador es un gestor en la medida en que conoce las características de cada medio, sus alcances y uso, para lograr diseñar un mix de medios tanto virtuales como tradicionales (en caso de ser necesario), que compaginen sus virtudes para apovar la 
Cuando la organización piensa en la necesidad de una Intranet para mejorar la comunicación entre sus miembros, debe tener en cuenta varios aspectos para su creación y mantenimiento, en el espacio virtual.

Los aspectos más importantes a tener en cuenta en la etapa de planificación de la Intranet son:

\section{Compromiso decidido de todos los miembros de la organización en su creación y mantenimiento}

Debe entenderse que la web es una potente herramienta de comunicación relacional, que genera beneficios a todos, por lo que se debe tener muy claro el rol que está cumple dentro del plan de comunicaciones de la organización.

\section{El WebMaster}

Es necesario nombrar a un responsable con autoridad y directrices claras que gestione y de mantenimiento a la web. El webmaster es el encargado de automatizar el concepto de negocio de la empresa, contando con un conocimiento amplio de la dinámica de la organización, pues debe llevar esa experiencia física al mundo virtual, de ahí la necesidad de contar con información muy amplia sobre la naturaleza de la institución, su filosofía, qué quiere proyectar, qué busca con la Intranet, qué papel tiene en el mix de medios internos.

Debe tener información sobre las necesidades de comunicación y relacionamiento que la Intranet quiere llenar respecto a sus objetivos comunicacionales. Aquí es muy importante el trabajo interdisciplinario con el encargado de comunicaciones internas y otros profesionales que se requieran de acuerdo a los objetivos del negocio.

\section{Escuchar a los miembros de la organización (directivos y empleados)}

Se debe recoger las inquietudes y necesidades de cada categoría de usuarios, para llevar esta información al diseño de la herramienta, en caso contrario, será una pérdida de recursos. Todos los miembros de la organización deben sentirse y ser parte del proyecto. Todo cambio genera resistencia y sólo si el empleado entiende las bondades del sistema para su labor profesional, podrá integrarse, aceptar y optimizar el uso de la Intranet para la institución.

El empleado es quien da las pautas para desarrollar los contenidos y funciones que la Intranet debe tener, para cumplir con las expectativas institucionales, pues no todos trabajan de la misma forma ni tienen las mismas necesidades.

\section{Conocimiento del modelo de comunicación institucional}

Es muy importante, comenzar por conocer a profundidad el modelo comunicacional que se vive en la institución. La Intranet debe responder al flujo comunicacional propio y no creer que la herramienta por sí misma, optimiza y mejora la 
comunicación interna. Esta herramienta debe ser parte del mix de medios definidos en el plan estratégico de comunicaciones.

\section{Pluralidad idiomática}

Es vital tener en cuenta que en ocasiones puntuales, la Intranet sobre todo de multinacionales pueden ser soporte para varios países, de ahí que deba tener la opción de distintos idiomas para facilitar a todos sus empleados, sin distingo idiomático el acceso a toda la información.

\section{Niveles de seguridad}

Definir los niveles de acceso de seguridad de acuerdo a los contenidos y usuarios de estos, para garantizar que la información confidencial no se filtre o salga de los límites de la organización.

\section{Entrenamiento}

Ofrecer entrenamiento a los miembros de la organización que serán usuarios de la Intranet, además de crear soportes en línea y tutoriales, para facilitar el uso y navegación en el sitio. Este punto no sólo se debe tener en cuenta al inicio del proyecto, sino que debe mantenerse en el tiempo y además incluirse en el plan de inducción para los nuevos empleados.

\section{Promocionar a la Intranet}

Promocionar la existencia de la Intranet y generar tráfico, a través de otros canales que hagan parte del mix de comunicaciones de la organización, como pueden ser correos electrónicos, boletines electrónicos y físicos, papelería corporativa y material POP donde se incluya la dirección de la Intranet, entre otros medios de comunicación institucionales y marcarios.

Así mismo, generar actividades y procesos donde la información requerida para ello, sólo se encuentre en la Intranet, como una forma de generar tráfico en ella e ir creando el hábito de uso.

\section{Seguimiento de su efectividad}

Hacer seguimiento en cuanto a la efectividad que se está teniendo en cubrir las necesidades comunicacionales, así como las que se puedan estar generando a partir de su uso, como pueden ser nuevas secciones, tutoriales, acciones cruzadas, etc.

\section{Intranet alineada a la identidad corporativa}

La Intranet debe ser diseñada y orientada de acuerdo a la identidad corporativa, ya que ésta debe ser el reflejo y la expresión de la cultura y el clima organizacional, convirtiéndose en una ventana de comunicación fluida y adaptada a las necesidades de la organización, más allá de modelos predeterminados que quizá no representen la identidad real de la organización. 
Para complementar qué otros aspectos se deben tener en cuenta en la creación de Intranet, Inmaculada Postigo, añade:

En un sentido más concreto cualquier Intranet corporativa debe tener en cuenta otras cuestiones. En lo que a interacción en la comunicación se refiere, resaltar que el feedback inmediato que esta tecnología posibilita es un factor clave para la implicación del empleado en la cultura empresarial y para que perciba la intranet como una herramienta útil. Por lo tanto, una intranet no debe ser nunca únicamente un servidor de información, sino que ha de ir más allá y convertirse en un sistema comunicativo. Pero para que ello sea realidad, no sólo es básico la implantación de la intranet, sino que hay que provocar un cambio en las actitudes tanto de los empleados como de los mandos: los primeros han de ser incitados a expresar su opinión y los segundos habrán de acostumbrarse a atender a los requerimientos de los trabajadores e incluir entre sus tareas darles respuesta a sus iniciativas de manera rápida (ser accesibles y responder a las cuestiones que les planteen). (Postigo, 2004, p.5)

El Dircom, debe ser un punto de equilibrio entre la realidad organizacional, las políticas comunicacionales y la democratización de la información virtual, para que el sujeto no encuentre un vacío entre su representación como individuo y su expresión colectiva como organización. Deben buscarse espacios para que se logre la intersección entre las dos dimensiones.

Es un conocedor de la organización, su naturaleza y funcionamiento, lo que le permitirá comprender la situación real de la comunicación y su entorno, pues en ocasiones se cree que con diseñar múltiples herramientas tecnológicas y digitales se subsanan los errores y vacíos de información y comunicación, olvidando que en ocasiones hay miembros de la organización que no cuentan con los equipos necesarios para acceder a estos medios, no poseen la preparación y competencias para su uso o simplemente no tienen el interés en hacerlo, relegándolos al abandono y a la discriminación por su perfil tecnológico dentro de la institución.

Un estudio realizado por Chavarro y Puerto sobre la "Comunicación por Intranet y sus efectos en las redes sociales en la organización" brindan algunos lineamientos a tener en cuenta desde una perspectiva sicológica, donde se reconfirma la importancia de la interdisciplinariedad en la gestión de la comunicación digital.

Se aplicaron 48 entrevistas en profundidad, indagando percepciones, sentimientos y conceptos a gerentes de grandes, pequeñas y medianas empresas, usuarias y no usuarias de Intranet. Los resultados mostraron que (Chavarro \& Puerto, 2002):

a) La totalidad de los entrevistados consideran una mejoría en el tráfico y en los canales de comunicación, optimizando su desempeño laboral generando 
conceptos positivos acerca del sistema.

b) Un porcentaje alto lo considera un medio muy impersonal, que aunque posee ventajas, pierde el respaldo de la comunicación directa.

c) Concluyen que la Intranet no reemplaza la comunicación interpersonal, sino la manera de trabajar.

\subsection{La comunicación en las Universidades}

Vilaseca i Requena, opina que la organización flexible, tan extendida en el mundo empresarial es uno de los pilares vertebradores en la eficacia de las universidades y los centros de investigación. En este marco, la flexibilidad se convierte en un concepto estructuralmente fuerte y se define por tres elementos fundamentales (Vilaseca \&Requena, 2009):

a) Universidades con capacidad de articular redes interdisciplinarias de docencia e investigación (superación del concepto profesor universitario autosuficiente).

b) Universidades con capacidad de articularse en redes institucionales de docencia y de investigación (superación del concepto universidad autosuficiente).

c) Universidad con capacidad de articular conexiones con la actividad productiva relaciones universidad y empresa) (Vilaseca \&Requena, 2009).

Las nuevas tecnologías están llevando a que el rol del profesor como lo conocemos cambie y pase de ser un actor que ofrece los conocimientos a ser un orientador con sus alumnos, para que sean éstos los que generen sus propios conocimientos. Esto conlleva a que el profesor tenga que desarrollar un trabajo interdisciplinario con pedagogos, especialistas en sistemas y hasta gestores del cambio, para poder estar al ritmo de los estudiantes, ya que para las nuevas generaciones el uso de las TIC es un asunto ordinario, que hace parte de su vida cotidiana.

Pero este cambio no sólo se debe dar a nivel de las aulas, si no de la gestión universitaria en general, pues según algunos datos que presentaremos a continuación, las universidades tanto públicas como privadas aún presentan una nota de suspenso en cuanto a su labor comunicacional.

En el Seminario Regional sobre medios universitarios en América Latina y el Caribe, se presentaron las conclusiones de un estudio realizado en 2008, en las universidades peruanas y los datos son reveladores: la universidad peruana sea pública o privada no está dispuesta a hablar de su comunicación, mostrar sus estrategias o evidenciar sus soportes tecnológicos en caso que los tenga. 
Por un lado está la comunicación institucional y por otro la comunicación académica. La primera corre a cargo de las oficinas de prensa o imagen o comunicación de las universidades. La segunda la propician las escuelas, facultades o institutos universitarios. Entre una y otra hay a veces un divorcio que no permite consolidar la voluntad comunicativa de la universidad. Esta dispersión puede ser percibida como positiva por las autoridades académicas y no así por las oficinas centralizadas de comunicación de las universidades.

La idea de medios universitarios se reduce al interés por piezas impresas como revistas, boletines o periódicos. Su periodicidad no es regular y el tiraje apenas cubre el mínimo de sus públicos internos. La radio universitaria se mimetiza con la comercial, lo que la obliga a generar discursos informales, sin características académicas. En el caso de las universidades públicas, la Ley de Transparencia de Perú ha obligado a generar páginas web que cumplan una "función informativa y educativa.

Se ha logrado ampliar los públicos y mejorar las cuotas de interacción entre universidades y los estamentos docentes, administrativos y estudiantiles así como con la comunidad" 3

Geovanny Orjuela, explica que "paradójicamente las universidades cuentan con facultades de comunicación y administración, pero sus modelos administrativos al parecer no aplican la teoría administrativa contemporánea, sino que aún mantienen modelos de gestión clásicos" (Orjuela, 2009).

En Colombia, en marzo de este año, se realizó un estudio en 35 universidades del país en el marco del encuentro sobre el Manejo de las comunicaciones en las universidades públicas, propiciado por el Ministerio de Educación Nacional y la Universidad Nacional de Colombia. Este estudio señala que el principal problema que afecta a estas instituciones es la falta de canales de doble vía, sus directivos carecen de competencias de liderazgo, la deficiencia en la comunicación entre las diversas áreas es importante, aunque la mayoría de estas universidades cuentan con Facultades de Comunicación Social. Sus modelos de comunicación dificultan el direccionamiento y logro de los objetivos institucionales.

El 50\% de los encuestados señalan que la incidencia de los problemas comunicacionales en los inconvenientes de gestión, es directamente proporcional entre ellos. En cuanto al clima organizacional, el $40 \%$ de los directores de comunicación consideraron que sí lo afecta directamente. Con este mismo porcentaje calificaron la incidencia de la comunicación interna en la "satisfacción del usuario".

\footnotetext{
${ }^{3}$ Seminario Regional sobre medios de comunicación universitarios en América Latina y el Caribe, pdf
} 
Desde otra perspectiva, Antonio Marín, Irener Trelles y Guadalupe Camarrón añaden que:

... teniendo en cuenta que aún cuando las universidades se validan cada vez más en el ámbito internacional, es la pertenencia a las comunidades regionales la que otorga a estas instituciones auténtico sentido, es necesario contribuir desde el punto de vista comunicacional a potenciar el papel de estas organizaciones como agentes del desarrollo local y a incrementar la vinculación de las mismas con las más diversas entidades de la sociedad civil.

Asimismo, deben ensayarse respuestas creativas frente a procesos novedosos derivados de la notable expansión de las tecnologías de la información y la comunicación TICs a nivel institucional.

En esta línea, en el plano de la comunicación interna, al trascender las fronteras regionales y nacionales se modifica la composición de la comunidad universitaria haciendo de los alumnos que cursan bajo esta modalidad, un nuevo segmento del estudiantado al que es necesario integrar. Igualmente, en el plano de la comunicación externa, se impone encarar tanto la consolidación de una imagen institucional propia como la promoción de estas nuevas ofertas académicas en un mercado educativo que se está globalizando y obliga a las universidades a competir con otros sistemas nacionales o regionales de formación y acreditación.

Por otro lado, es imprescindible resolver los inconvenientes que resultan de la extensión escasamente planificada y, por tanto, desordenada de las TICs hacia el interior de las instituciones". (Marín, Trelles y Zamarrón, 2005, p.40).

\subsection{La Formación en la Empresa}

Una Escuela de Negocios es una estructura académica de estudios, diferente a la de pregrado y postgrado, que responde a la demanda de formación de alto nivel para líderes y ejecutivos empresariales y sociales, por ello cuenta con unas características muy propias por lo que se considera importante, para efectos de este trabajo definir brevemente qué es la formación en la empresa, sus objetivos y beneficios. Pineda, citando a Goldstein, I.L. expone:

Formación en la empresa se define como la adquisición sistemática de habilidades, normas, conceptos y/o actitudes que conducen a una mejora de ejecución en el contexto laboral. Formación en la empresa puede definirse como el proceso que pretende eliminar las diferencias existentes entre aquello que un empleado puede ofrecer a partir de sus habilidades, experiencias $v$ 
Los objetivos de la formación a los miembros de la empresa son:

a) Ofrecer habilidades para realizar tareas más complejas y de mayor responsabilidad.

b) Actualizar los conocimientos y destrezas, para afrontar los avances que se producen en el entorno laboral.

c) Cubrir necesidades de desarrollo personal, a la vez que se prepara mejor para su desempeño profesional.

Los beneficios que genera la formación empresarial:

a) Ser más competitivos.

b) Reforzar sentido de pertenencia del empleado con su empresa, al ofrecerle la oportunidad de estudio y progreso.

c) Preparar al empleado para reaccionar ante el cambio y la incertidumbre.

d) Garantizar una mayor flexibilidad y capacidad de adaptación del propio equipo de trabajo.

e) Retención de sus empleados más valiosos y atracción del mejor capital humano que está en el mercado.

f) Formación de una fuerza laboral actualizada, motivada y eficaz. (Pineda, 2003)

La comunicación en las universidades es un tema para seguir trabajando, pues como agente generador de conocimiento y a partir del desarrollo de nuevos medios de comunicación, seguirá existiendo la necesidad de seguir investigando, desarrollando e innovando.

\subsection{Caso de estudio, la Escuela de Negocios en Venezuela ${ }^{4}$}

Esta Escuela de Negocios en Venezuela se encarga de desarrollar programas gerenciales para cargos medios y altos en las organizaciones públicas y privadas. Es una unidad que reporta directamente al Vicerrectorado Académico, diferente a la de pregrado y postgrado, que cuenta con unas características muy propias ya que busca ofrecer herramientas para el profesional especializado con una base teórica de innovación y actualidad.

Su misión es ofrecer al profesional de hoy la actualización y motivación que requiere para: maximizar su desempeño laboral, transformar su entorno organizacional, producir riqueza y generar bienestar social y ambiental.

\footnotetext{
${ }^{4}$ Toda la información institucional fue tomada de los documentos de creación de la ENV y a partir de entrevista personal con su Directora General
} 
Su visión, es desarrollar y producir la oferta internacional de actualización técnicoprofesional en gestión y transformación organizacional de mayor pertinencia, excelencia académica y calidad de servicio del mercado regional.

Su Filosofía: El modelo de gestión de la ENV está inspirado en la "Calidad del Servicio", entendiéndose como la satisfacción y superación de las expectativas del cliente y para lograrlo es necesario conocer qué aspira recibir el cliente cuando se dirige a la ENV, por lo tanto el eje de todas las actividades que planifica y su modo de llevarlas a cabo es a partir del conocimiento profundo acerca de qué aspira recibir el cliente cuando se dirige a la ENV:

a) Acreditación académica.

b) Una oferta variada, oportuna.

c) Actividades forativas y de actualización profesional de excelente calidad académica.

d) Facilitadores experimentados capaces de combinar la teoría y la práctica profesional.

e) Lograr una mejora sustancial de su rendimiento y progreso profesional.

f) Precios razonables y facilidades de pago.

g) Una infraestructura adecuada para el aprendizaje de adultos, de fácil de acceso.

h) Un paquete integral de servicios.

Para poder cumplir estas expectativas, la ENV está estructurada con base en un criterio mixto:

a) Por producto para el caso de las unidades de negocio: Adiestramiento presencial - Adiestramiento virtual - Adiestramiento corporativo y Programas internacionales.

b) Por funcionamiento para las unidades de staff: Administración Financiera Automatización de Procesos - Mercadeo y Ventas - Servicios Generales.

Todas las unidades de negocio manejan los procesos inherentes al producto de adiestramiento que comercializan y del que son responsables de principio a fin.

Esto abarca desde el análisis del entorno, del mercado y de las tendencias, pasando por el diseño de la oferta, la identificación de docentes, la fijación de matrículas, el control de las reservaciones e inscripciones, hasta la ejecución de la actividad propiamente dicha incluyendo la atención al participante y al docente, el registro de asistencias, las evaluaciones -si las hubiere-, la emisión de certificados una vez que finaliza la actividad y la evaluación del desempeño docente.

En resumen cada coordinador es el responsable "integral" y el garante de la calidad de todas las actividades que organiza. Cada unidad de negocio utiliza tarifas e índices 
Este marco de referencia les permite realizar los estudios de factibilidad financiera de cada actividad, calcular matrículas y negociar honorarios con los profesores (dentro de los rangos preestablecidos). Del mismo modo cada unidad de negocio utiliza formatos estandarizados para elaborar los estados de resultados de cada actividad realizada.

Trimestralmente cada coordinador elabora un informe de resultados con base en indicadores de gestión, tales como: actividades programadas vs efectivamente realizadas y de éstas vs suspendidas, tasa de abandono, valor promedio de la hora de instrucción, número de estudiantes inscritos, facturación bruta, costos, ingresos netos y tasa de rendimiento.

El estudio de factibilidad permite el establecimiento del punto de equilibro de cada actividad, o número mínimo de participantes inscritos que permite la cobertura de todos los costos, incluyendo la marca corporativa y la obtención del margen de rendimiento mínimo deseable.

Después de establecer la matricula y la programación de cada actividad se definen los modos, tiempos, medios y segmentos de promoción:

Actualmente la ENV, está ubicada en el centro financiero de Caracas y cuenta con 24 miembros, divididos en 10 profesionales (coordinadores de unidades de negocio y unidades de staff), 7 empleados (administrativos, de vigilancia y mantenimiento) y 7 pasantes que estudian en diferentes Facultades de la misma Universidad.

\subsection{Resultados de la investigación}

\subsection{La comunicación interna en la ENV}

\subsection{Opinión de los miembros administrativos}

A partir de la encuesta realizada a 14 de los 20 miembros administrativos de la ENV, se obtuvo información que sirve como un primer acercamiento al estado de la comunicación, con el fin de contextualizar la Intranet (SAAI) en ella. Los resultados fueron los siguientes:

La comunicación interna es calificada como regular en un $42,86 \%$, seguida por un $35,71 \%$ que la considera buena y un $21,43 \%$ excelente. Al preguntarles por los problemas de comunicación en la ENV, las respuestas fueron:

a) Deficiencia en la comunicación en los horarios rotativos, con el correo ha mejorado, sin embargo hay muchos vacíos y cruce de información, por lo que es vital mejorar el SAAI.

b) El mail es el medio de comunicación privado favorito con 56,25\%, seguido por $25 \%$ con el teléfono fijo y el $12,50 \%$ con el móvil. 
c) El cara a cara, cuenta con $21,43 \%$ de aceptación. Se hizo énfasis en recuperar las reuniones semanales o quincenales, así como realizar algunos talleres o actividades que desarrollen y refuercen las competencias comunicacionales individuales e interpersonales.

\subsection{Opinión de las directivas de la ENV}

Al entrevistar a las dos máximas directivas (Directora General y Coordinadora de Mercadeo), se encontró que:

En sus 12 años de existencia la ENV no había contado con un plan de comunicaciones, ni con un coordinador de comunicaciones y sólo hasta hace 2 años se ha diseñado un Plan de Mercadeo, el cual ha incluido entre sus medios de comunicación a la Intranet, en primer término para alimentar la página web y por otro lado para: proteger la data, articular la información y procesos de las distintas unidades. Para tener un solo sistema y así concentrar y homologar toda la información y todo el trabajo que se genera. Conectar las distintas etapas del trabajo (académica, logística, administrativa) y que todos accedan a todo el trabajo desde una misma fuente.

Al no existir un plan de comunicaciones, ni tampoco unos lineamientos de comunicación interna claros, el SAAI es un medio que se utiliza sólo por algunos de los empleados de la ENV, sin tener muy claro su objetivo como herramienta comunicacional y por sí sólo no da resultados óptimos, para coadyuvar en el mejoramiento de la comunicación interna.

El mayor inconveniente de comunicación radica en los diversos turnos de trabajo que se tienen y la inexistencia de un medio que les permita cruzar y compartir la información que se genera en los diferentes horarios. El verbatim lo evidencia "Cuando inician los cursos el cruce de información es grave y el cliente lo detecta". El (SAAI), fue puesto en funcionamiento hace aproximadamente 2 años y en este tiempo no se ha hecho una evaluación de su efectividad.

La Intranet presenta deficiencias en todas las categorías que se identificaron para analizar, lo que demuestra que el SAAI no está generando lo que se esperaba de él, porque desde su concepción no se llevó al diseño, las necesidades puntuales de cada área. Esto se puede ver cuando de las 4 áreas del negocio, 3 no la utilizan; y de las 4 áreas de staff, 2 no la usan, por no encontrar la información que requieren o tenerla muy limitada, para su trabajo efectivo, resultando subutilizado y generando un gasto en su "mantenimiento".

Dos casos emblemáticos de esta situación son: área de Finanzas que aunque tiene un ícono en la Intranet no está funcionando y no le permite cruzar la información entre los inscritos, pagos realizados y cuentas por cobrar, generándoles reprocesos al tener 
En el caso de la Coordinación de Programas Internacionales, deben trabajar con el programa Banner (notas, expedientes, etc.) del Postgrado de la Universidad y la Intranet no les resulta útil.

\subsection{Análisis de la Intranet (SAAI)}

El SAAI no cuenta con 7 de las 9 variables que se consideran mínimas para una Intranet actual. Éstas son: presentación o saludo de la dirección, segmentación de públicos, boletines electrónicos, sección de noticias de la ENV y de la Universidad.

No hay opciones de ocio, no se puede personalizar y la información está desactualizada, lo que resulta muy grave sobre todo porque su objetivo de uso actual se centra en las reservaciones e inscripciones para los cursos y programas que se ofrecen al público general y empresarial de Venezuela.

En cuanto a la usabilidad, el sistema sí está dividido en categorías, la marca visual de la ENV y de la Universidad, aparecen al abrir cada categoría y cada sección del sitio tiene un título que identifica el contenido de la pantalla, aunque a veces se pierde por el tamaño de la letra.

La autonomía y control se cumple al poder volver fácilmente a menús previos, abandonar el sistema fácilmente y navegación fácil, pero no tiene mapa del sitio, motor de búsqueda y no ofrece varias rutas de navegación.

En cuanto a la legibilidad cumple con el tipo de letra en casi todo el sistema, las frases y párrafos cortos, los íconos son visualmente claros y el contraste entre texto y fondo es equilibrado y permite la lectura.

La consistencia es débil es el aspecto de los comandos que no se utilizan de la misma manera en todo el sistema, ni respeta las convenciones como el sombreado de los vínculos visitados, generando retrasos en la navegación. En cambio sí hay una identificación de páginas y secciones uniforme y se distingue claramente cada página.

Acerca de la eficiencia, el tiempo de carga del home es entre 6 y 15 segundos, lo que para esta herramienta de trabajo es un poco lento. Se necesitan 2 click para encontrar la información y la estructura del sitio es simple. La interfaz no presenta varias formas de buscar la información, ni da posibilidades de descargas, envío por mail y sólo el $5 \%$ de los documentos se pueden imprimir.

La interactividad es nula, pues no cuenta con foros, chat, blog, RSS, twitter, facebook, ni ningún otro medio, ni siquiera un tutorial o ayuda en línea en caso de presentarse dudas para la navegación v búsaueda de información. 
Al revisar la información especializada propia del sector educativo la deficiencia es evidente, pues no presenta información corporativa (institucional, manuales de funciones, de procesos, directorios, memorias, tutoriales, modelos y formatos para presentar propuestas), ni información sobre profesores, link con la Universidad, ni sección de preguntas y respuestas. Sólo cuenta con información limitada de los cursos en cuanto a personas interesadas, reservadas e inscritas y algunas bases de datos, pero no cuenta con información propia de cada curso y programa, como objetivos, dirigido a, contenidos, montos de inscripción, etc. Para ver esta información debe salir del SAAI y entrar en la página web de la ENV.

Se hizo una revisión de lo que debiera tener como sala de prensa virtual (archivo de notas de prensa, comunicados, fotografías o videos) y sólo cuenta con una agenda de eventos, entendida como la programación de los cursos y programas.

\section{CONCLUSIONES}

Al no existir un plan de comunicaciones, ni tampoco unos lineamientos de comunicación interna claros, el SAAI es un medio que se utiliza por algunos de los empleados, sin tener muy claro su objetivo como herramienta comunicacional y por sí sólo no dará resultados óptimos, para coadyuvar en el mejoramiento de la comunicación interna.

La comunicación interna es calificada en un alto índice como regular, lo que revela deficiencias que no se relacionan directamente con la Intranet, pues la situación que se ha diagnosticado de esta herramienta es el reflejo de la situación comunicacional de la institución.

El mayor inconveniente de comunicación radica en los diversos turnos de trabajo que se tienen y no la inexistencia de un medio que les permita cruzar y compartir la información que se genera en los diferentes horarios. El verbatim lo evidencia "Cuando inician los cursos el cruce de información es grave y el cliente lo detecta".

En cuanto a medios favoritos son: mail y el teléfono fijo y para su trabajo en la institución el que prefieren es de nuevo el mail, lo que demuestra su interés por los medios digitales. Además les interesa mucho recuperar espacios para el cara a cara y esto se evidencia en que es la propuesta que más se repite a la hora de pedirles soluciones en búsqueda de una comunicación interna más efectiva y en menor escala pero no por esto menos importante proponen hacer talleres para mejorar la comunicación interpersonal.

La Intranet presenta deficiencias en todas las categorías que se identificaron para analizar, lo que demuestra que el SAAI no está generando lo que se esperaba de él, porque desde su concepción no se llevó al diseño las necesidades puntuales de cada 
Esto se puede ver cuando de las 4 áreas del negocio, 3 no la utilizan; y de las 4 áreas de staff, 2 no la usan, por no encontrar la información que requieren o tenerla muy limitada, para su trabajo efectivo, resultando subutilizado y generando un gasto en su "mantenimiento".

El Sistema Automatizado de Administración Integral no cuenta con 7 de las 9 variables que se consideran mínimas para una Intranet actual. Éstas son: presentación o saludo de la dirección, segmentación de públicos, boletines electrónicos, sección de noticias, no hay opciones de ocio, no se puede personalizar y la información está desactualizada, lo que resulta muy grave sobre todo porque su objetivo de uso actual se centra en las reservaciones e inscripciones para los cursos y programas que la ENV ofrece al público general y empresarial de Venezuela.

En cuanto a la usabilidad y legibilidad son las variables que presentan menos deficiencias. En autonomía y control no cuenta con mapa del sitio, motor de búsqueda y no ofrece varias rutas de navegación.

La consistencia es débil y en eficiencia su mayor problema es no tener la posibilidad de descargas, envío por mail y muy pocos documentos se pueden imprimir, lo que afecta en alto grado la rutina de trabajo de los usuarios.

La interactividad es nula y al revisar la información especializada que debiera tener para el sector educativo la deficiencia es evidente, pues no presenta información ninguna información corporativa: filosofía, manuales de procesos y operativos.

Se hizo una revisión de lo que debiera tener como sala de prensa virtual y sólo cuenta con una agenda de eventos, entendida como la programación de los cursos y programas. No presenta archivo de notas de prensa, fotografías o videos.

El SAAI es un recurso sub-utilizado se está perdiendo muchas posibilidades para mejorar la condición de la comunicación interna, así mismo se ha convertido en una herramienta ineficiente, pues actualmente sólo se utiliza para revisar el status de reservaciones y postulaciones, confirmación de interesados en cursos y programas, revisar cronogramas si hay aulas.

Es interesante revisar que aunque un alto porcentaje considera al SAAI útil, al cruzar las respuestas y opiniones que enriquecen cada pregunta, se denota que es más un sentido de pertenencia a la organización lo que les lleva a pensar que les es útil, pero es más efecto emocional que real, pues los usuarios que no la usan se muestran resignados a su situación. 
El SAAI no se puede ver como un medio de mejoramiento de la comunicación interna en la ENV, porque en sí mismo presenta muchas deficiencias, pero las directivas no conocen la situación real de lo que viven en su jornada laboral los pasantes y empleados. De ahí que las directivas sólo lo vean como un medio que se debe "perfilar" nada más. Un verbatim arroja luces acerca del uso y efectividad de la Intranet: "Tiene que ver con el uso no con la herramienta, no nos estamos anticipando al crecimiento del departamento, lo estamos persiguiendo, cada solución se queda corta". Y se complementa con "En la etapa de diseño se nos preguntó qué necesitábamos, pero en la práctica eso no se tuvo en cuenta".

No es una herramienta que agregue valor a la gestión de las áreas que se desarrollan en la ENV, pues no los ayuda a organizar su trabajo ni a ser más productivo.

Actualmente el SAAI cuenta con un espacio denominado envío de mensajes, pero es muy poco utilizado. Le falta promoción entre los usuarios y con un sistema de alarma para nuevos mensajes, pudiera ser una opción para registrar las novedades en los tres turnos.

La ENV debe definir inicialmente sus variables comunicacionales y establecer cuál es su modelo comunicacional, para luego diseñar medios que los apoyen en su plan de medios, pero mientras se diseñen medios sin tener un fin, ni una visión estratégica de la comunicación, no se logrará mejorar el proceso en sí mismo y las herramientas por más que sean de última tecnología no lograrán cumplir con su fin como medio digital activador de soluciones funcionales a nivel comunicacional, generando beneficios para la organización.

Se puede concluir que la Intranet ó SAAI, no está cumpliendo las funciones inherentes a ésta, por lo tanto no se puede denominar Intranet, ya que sólo es un administrador limitado de contenidos, que realmente agrega poco valor a la gestión administrativa y académica de la institución.

\section{REFERENCIAS}

Escobar Sarría, J. (2007). Nuevas formas de planear estratégicamente la organización y la comunicación. Ponencia presentada en el VI Simposio Latinoamericano de Comunicación Organizacional. Universidad Autónoma de Occidente en Cali. Colombia.

Day, G. \& Hubbard, K. J. (2004). Beneficios y verdades del contacto digital. Revista Gestión, 1 de febrero-marzo.

Llano, S. (2007). Dimensión estratégica en la Comunicación Organizacional de las tecnologías de información v comunicación v los nuevos medios. En Comunicación 
Núñez Noda, F. (2009). Comunicación digital y web. Caracas: Editorial CEC, S.A.

Olaya Chavarro, D. \& Puerto Velandia, J. (2002). Comunicación por Intranet y sus efectos en las redes sociales en la organización. Trabajo de grado de Licenciatura en Sicología dirigida por la Dra. Silvia Martí, no publicado, Universidad de La Sabana, Bogotá, Colombia.

PINEDA, Pilar (2003): Auditoría de la Formación. Ediciones Gestión 2000. Barcelona.

Postigo, I. (2009, julio). Intranet como herramienta de comunicación interna. Módulo dictado en el Doctorado en Comunicación Organizacional. Universidad de Málaga Universidad Mayor de Chile. Apuntes propios.

Postigo, I. (2004). La Intranet al servicio de la comunicación vs. La comunicación al servicio de la Intranet. Revista de la Escuela de Comunicaciones UVM, 4(4).

Campos Freire, F. (2008). Las Redes Sociales trastocan los modelos de los medios de comunicación tradicionale. Revista Latina de Comunicación Social, 063(11).

Recuperado el 12 de julio de 2010, de www.ull.es/publicaciones/latina/_2008/23_34_Santiago/Francisco_Campos.html

Comuzi, I. (2002). Tecnologías de la comunicación en la formación docente.

Revista Comunicar, 019 de octubre. Recuperado el 21 de julio de 2010, de http://redalyc.uaemex.mx/redalyc/src/inicio/ArtPdfred.jsp?iCve=15801923

García Jiménez, L. (2008). Las Ciencias de la Comunicación a laluz de las nuevas tecnologías: Retos para una disciplina en la incertidumbre. Global Media Journal, 5. Recuperado el 15 de julio de 2011

http://gmje.mty.itesm.mx/lascienciasdelacomunicacion.pdf

Marín Ruíz, A.; Trelles Rodríguez, I. \& Zamarrón Garza, G. (2005). MASS MEDIA Y UNIVERSIDAD. El reto de la comunicación en las universidades en Diálogo Iberoamericano. Granad a: Universidad de Granada.

Murelaga Ibarra, J. (2005). Breve Reflexión de la Sociedad Tecnologizada actual. Tecnología Digital, Individuo, Globalización e Internet. Revista Latina de Comunicación Social, 59 de enero-junio. Recuperado el 11 de agosto de 2010 , de http:/ / www.ull.es/publicaciones/latina/200502murelaga.pdf.

Orjuela, G. (2007).Los problemas de comunicación en las universidades públicas generan un $50 \%$ de sus dificultades de osctión Resunerado el 1 de sentiemhre de 7009 de 
www.gestiopolis.com/administracion-estrategia/dificultades-en-la-gestion-decomunicacion.html

Vilaseca I Requena, J. Tecnologías de la información y la comunicación en las universidades. Recueprado el 1 de septiembre de 2009, de www.uoc.edu/web/esp/articles/vilaseca/tic.html .

Estudio regional: Caso Perú. Ponencia presentada en el Seminario regional de medios universitarios en América Latina y el Caribe. Recuperado el 1 de septiembre de 2009, de www.seminarios.pe/medios.

\section{Sandra Orjuela-Córdoba}

Comunicadora Social y Periodista de la Universidad de La Sabana (Colombia), especialista en Sociología del Consumo e Investigación de Mercados -Universidad Complutense de Madrid - Cursos de Máster en Comercio Exterior y Marketing Internacional - Universidad Politécnica de Madrid. Candidata a Doctora en Comunicación Organizacional en la Universidad de Málaga. Con experiencia de diecinueve años a nivel directivo y desde la consultoría en el campo de la Comunicación Organizacional, el Marketing y la Responsabilidad Social para diversas empresas en Iberoamérica, en diversos sectores de la industria y la academia. 